\title{
Characterization of the Corpus Callosum Morphology in Healthy Sudanese Adults using MRI
}

\author{
Alnour Badawi, $\mathrm{MSc}^{1}$; Caroline Edward Ayad, $\mathrm{PhD}^{2}$; Mogahid M.A. Zidan, $\mathrm{PhD}^{3}$; \\ Ikhlas Abdalaziz, $\mathrm{PhD}^{2}$; Batil Alonazi, $\mathrm{PhD}^{4}$; Mustafa Z. Mahmoud, $\mathrm{PhD}^{4 *}$; \\ Mohamed Yousef, $\mathrm{PhD}^{5}$; Lubna Bushara, $\mathrm{PhD}^{6}$; Mohamed Adam, $\mathrm{PhD}^{7}$ \\ ${ }^{I}$ Medical Imaging Technology Department, Al-Ghad International College for Applied Medical Science, \\ Najran, Saudi Arabia \\ ${ }^{2}$ Diagnostic Technology Department, College of Medical Radiological Sciences, Sudan University of \\ Science and Technology, Khartoum, Sudan \\ ${ }^{3}$ Medical Imaging Technology Department, Al-Ghad International College for Applied Medical Science, \\ Abha, Saudi Arabia \\ ${ }^{4}$ Radiology and Medical Imaging Department, College of Applied Medical Sciences, \\ Prince Sattam bin Abdulaziz University, Al-Kharj, Saudi Arabia \\ ${ }^{5}$ Radiologic Sciences Program, Batterjee Medical College, Jeddah, Saudi Arabia \\ ${ }^{6}$ Department of Medical Imaging and Radiation Sciences, College of Applied Medical Sciences, \\ University of Jeddah, Jeddah, Saudi Arabia \\ ${ }^{7}$ Department of Radiological Sciences, College of Applied Medical Sciences, King Khalid University, \\ Abha, Saudi Arabia
}

\begin{abstract}
Background: The aim of this study was to characterize the morphological differences of the corpus callosum (CC) concerning gender- and age-related variations, and establish reference values of the CC measurements for Sudanese participants using magnetic resonance imaging (MRI).

Methods and Results: MRI scans from 196 males and 189 females, ranging in age from 18 to 84, were reviewed. Only MRI studies without pathological findings were included in the analysis. The following measurements were done: the longitudinal dimensions of the brain, CC, the genu of $\mathrm{CC}$, the splenium of CC, as well as craniocaudal height of the body of CC. Callosal longitudinal dimensions were measured using the Witelson division method. The longitudinal dimension of the genu was found to be larger in females $(11.12 \pm 1.54 \mathrm{~mm})$ than in males $(10.73 \pm 1.75 \mathrm{~mm})(P=0.021)$. The longitudinal dimension of the brain was greater in males $(161.6 \pm 7.2 \mathrm{~mm})$ than in females $(159.3 \pm 7.6 \mathrm{~mm})(P=0.002)$. There was a weak statistically significant direct correlation between age and $\mathrm{CC}$ ratio $(\mathrm{r}=0.271, P<0.05)$.

Conclusion: The results revealed that only the genu length showed sexual dimorphism and was larger in females than in males. This study showed variabilities regarding the CC dimensions in Sudanese participants compared to the Caucasian, Indian, and Japanese population.(International Journal of Biomedicine. 2020;10(3):215-220.)
\end{abstract}

Key Words: corpus callosum $\bullet$ gender $\bullet$ age $\bullet$ magnetic resonance imaging $\bullet$ Sudanese population

\section{Abbreviations}

APL, anterior-posterior length; CC, corpus callosum; CCI, corpus callosum index; MRI, magnetic resonance imaging

\section{Introduction}

The corpus callosum (CC) is unique to placental mammals in the brain structure that connects the right and left hemispheres.
It consists of approximately 200 million neural fibers and is responsible for the interhemispheric transfer of information and higher-order cognition. ${ }^{(1)}$ The most parsimonious explanation for callosal evolution is that it arose to facilitate long-distance 
integration within large brains. ${ }^{(2)}$ Callosal fibers are first found in human embryos at 10-11 weeks of gestation, and by 12-13 weeks, a rudimentary callosal plate can be seen. ${ }^{(3)}$ It connects the non-olfactory areas of the right and the left cerebral cortex. Initially, it forms a small bundle in the lamina terminals. As a result of the continuous expansion of the neopallium, however, it extends first anteriorly and then posteriorly, arching over the thin roof of the diencephalon. ${ }^{(3)}$ The CC first enlarges caudally then develops rostrally. Myelination occurs relatively slowly over the lifespan, with the process completing in puberty.(4) Myelination progresses caudally to rostrally, much as the CC develops, from the splenium to the genu and rostrum. It begins to develop around the 12th week of gestation and matures through a complex process of neuronal migration, development, and eventual neuronal pruning. ${ }^{(1,4)}$ By week 20, the CC can be seen on a sonogram or fetal MRI. ${ }^{(4)}$ Although the CC may be considered fully developed by around age, as with most neural structures, it likely continues to change over the lifespan. ${ }^{(5,6)}$

The majority of the literature has found that outcomes are better for individuals with dysgenesis of the CC when compared to individuals with complete agenesis of corpus callosum, though there is some conflicting evidence. Moderate evidence suggests that the presence of the anterior commissure which consists of the rostrum and the genu correlates with better cognitive and/or developmental functioning, though very few child studies have documented the degree of dysgenesis or which segments of the CC. ${ }^{(7,8)}$

The variability of the adult $\mathrm{CC}$ may vary with gender and age. There are, however, little data on the morphology of the CC in the Sudanese population. The purpose of this study was to investigate the relationship between morphometric CC measurements, age, and gender and establish $\mathrm{CC}$ reference values in Sudanese adults using MRI.

\section{Materials and Methods}

This study was approved by the local ethics committee and the Institutional Review Board (IRB) of the Faculty of Radiology, University of Medical Sciences and Technology (UMST), Khartoum, Sudan. A group of 385 participants (196 males and 189 females), presenting at the MRI units of AlZytouna Specialized Hospital, Royal Care Hospital, Al-Ribat Teaching Hospital, Antalya Hospital, Dar Al-Elaj Specialized Hospital, Fedail Hospital, and Al-Amal Hospital (Khartoum, Sudan), were recruited between March 2015 and August 2015 for this descriptive study. A disclaimer of the informed consent form was conceded as per institutes rules. Participants who gave data of a formerly known CC or the history of any neurological disease were excluded.

MRI brain examinations were conducted on a 1.5 Tesla MRI machine (Toshiba Medical Systems, Tokyo, Japan) at the Al-Zaytouna Specialist Hospital, Dar Al-Elaj Specialized Hospital, and Royal Care Hospital. A 1.5 Tesla MRI unit (Siemens Medical Systems, Munich, Germany) was used to examine participants at Fedail Hospital and Al-Ribat teaching hospital. In addition, a 1.5 Tesla MRI unit (Philips Medical Systems, Amsterdam, Netherlands) and (GE Healthcare Medical Systems, Chicago, United States) was used in Al-
Amal Hospital and at Antalya Hospital, respectively.

Each patient was scanned supine on the examination couch with his or her head within the head coil. The head is adjusted so that the interpupillary line is parallel to the couch. The brain MRI protocol included the following imaging planes: a) sagittal $\mathrm{T}_{1}$-weighted images, $\mathrm{b}$ ) axial $\mathrm{T}_{1}$-weighted images, c) axial $\mathrm{T}_{2}$-weighted images, d) axial fluid-attenuated inversion recovery (FLAIR) protocol, and e) coronal $\mathrm{T}_{2}$ weighted images. Slice thickness/interslice gap of $5 / 1.5 \mathrm{~mm}$ and matrix size of $256 \times 192$ were used.

In this study, we measured the longitudinal and vertical dimensions of the corpus callosum and its various parts. For each case, using a mid-sagittal view of the cerebral hemispheres, the following global dimensions were measured ${ }^{(2,9,10)}$ (Fig.1.): $\mathrm{AB}$ - Longitudinal dimension of the brain from the frontal to the occipital pole (anterior-posterior length (APL) of the brain); $\mathrm{EZ}$ - Length of CC from the most anterior point of the genu to the most posterior point of the splenium. Using the Witelson division method, the longitudinal dimensions of the genu $(\mathrm{E} / 3)$, and splenium (E/5) were measured. The width of the $\mathrm{CC}$ middle portion was considered as the body dimension. ${ }^{(11)}$

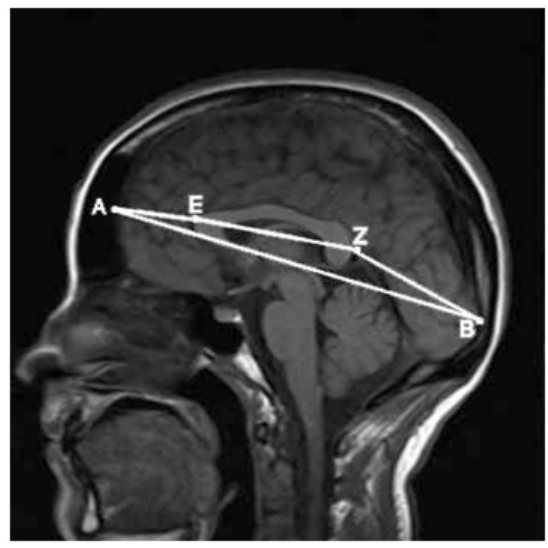

Fig 1. Brain MRI image demonstrates the measured dimensions.

A - Frontal pole of the brain; B - Occipital pole of the brain; $E$ - Genu of CC; $Z$ - Splenium of $C C^{(9)}$

Each image was recalled from the MRI computer's hard disk; brightness and contrast were set to approximate images used for clinical evaluation; the area of the $\mathrm{CC}$ was computed with the standard MRI software.

The obtained data were analyzed using the standard Statistical Package for the Social Sciences (SPSS Inc., Chicago, IL, USA) version 20. Continuous variables were presented as the mean, standard error of the mean (SEM), standard deviation (SD). For data with normal distribution, inter-group comparisons were performed using Student's t-test. Pearson's correlation coefficient (r) was used to determine the strength of the relationship between the two continuous variables. A probability value of $P<0.05$ was considered statistically significant.

\section{Results and Discussion}

A total of 385 Sudanese individuals participated in the study, aged from 18 to 84 years, with a mean of $41.7 \pm 18.6$ 
years. Of the 385 participants, there were 196(50.9\%) males (mean age of $44.9 \pm 19.37$ years) and 189(49.1\%) females (mean age of $38.4 \pm 17.19$ years).

$\mathrm{CC}$ morphometric measurement parameters are shown in Table 1.The study revealed that the mean values for the longitudinal dimensions of the $\mathrm{CC}$, genu, splenium, and body width were $74.23 \pm 5.12 \mathrm{~mm}, 10.92 \pm 1.6 \mathrm{~mm}, 10.92 \pm 1.47 \mathrm{~mm}$, and $5.43 \pm 0.89 \mathrm{~mm}$, respectively, while the mean value for for the longitudinal dimension of the brain was $160.5 \pm 7.5 \mathrm{~mm}$.

Table 1.

Descriptive statistics of study variables $(n=385)$

\begin{tabular}{|l|c|c|c|c|}
\hline \multicolumn{1}{|c|}{ Variables } & Minimum & Maximum & Mean & SD \\
\hline Splenium dimension $(\mathrm{mm})$ & 6.1 & 16.9 & 10.92 & 1.47 \\
\hline Genu dimension $(\mathrm{mm})$ & 4.4 & 15.6 & 10.92 & 1.6 \\
\hline Body dimension $(\mathrm{mm})$ & 2.3 & 7.9 & 5.43 & 0.89 \\
\hline Length of CC (mm) & 59.8 & 86.6 & 74.23 & 5.12 \\
\hline APL of the brain $(\mathrm{mm})$ & 139 & 183 & 160.5 & 7.5 \\
\hline Age & 16 & 84 & 41.76 & 18.60 \\
\hline
\end{tabular}

Differences between males and females with their levels of significance are summarized in Table 2. We found that the genu length was greater in females $(11.12 \pm 1.54 \mathrm{~mm})$ than in males $(10.73 \pm 1.75 \mathrm{~mm})(P=0.021)$. Our findings were not similar to the results obtained by Mourgila et al. ${ }^{(2)}$ for the Greek population and Takeda et al. ${ }^{(12)}$ for the Japanese population. In addition, there were no statistically significant gender-related differences for the longitudinal dimensions of the $\mathrm{CC}$ and corpus callosal splenium, as well as for the corpus callosal body dimensuin. Our findings were not similar to the results obtained by Suganthy et al. ${ }^{(13)}$ for the Indian population. There was a statistically significant gender-related difference for anterior-posterior length (APL) of the brain: 159.3 $\pm 7.6 \mathrm{~mm}$ in females and $161.6 \pm 7.2 \mathrm{~mm}$ in males $(P=0.002)$ (Table 2$)$.

Table 2.

Dimensions of CC in the Sudanese participants according to gender

\begin{tabular}{|l|c|c|c|c|c|c|}
\hline \multicolumn{1}{|c|}{ Variables } & Gender & $\mathrm{n}$ & Mean & SD & SEM & $P$-value \\
\hline \multirow{3}{*}{ Age (yrs) } & Male & 196 & 44.94 & 19.37 & 1.38 & \multirow{2}{*}{0.001} \\
\cline { 2 - 7 } & Female & 189 & 38.47 & 17.19 & 1.25 & \\
\hline \multirow{3}{*}{ Genu dimension (mm) } & Male & 196 & 10.73 & 1.75 & 0.12 & \multirow{2}{*}{0.021} \\
\cline { 2 - 7 } & Female & 189 & 11.12 & 1.54 & 0.11 & \\
\hline \multirow{3}{*}{ Body dimension (mm) } & Male & 196 & 5.38 & 0.95 & 0.06 & \multirow{2}{*}{0.270} \\
\cline { 2 - 7 } & Female & 189 & 5.48 & 0.82 & 0.05 & \\
\hline \multirow{3}{*}{ Splenium dimension (mm) } & Male & 196 & 10.84 & 1.46 & 0.10 & \multirow{2}{*}{0.316} \\
\cline { 2 - 7 } & Female & 189 & 10.99 & 1.47 & 0.10 & \\
\hline \multirow{3}{*}{ Length of CC (mm) } & Male & 196 & 74.58 & 4.87 & 0.34 & \multirow{2}{*}{0.168} \\
\cline { 1 - 6 } & Female & 189 & 73.86 & 5.35 & 0.38 & \\
\hline \multirow{2}{*}{ APL of the brain (mm) } & Male & 196 & 161.6 & 7.2 & 0.50 & \multirow{2}{*}{0.002} \\
\cline { 1 - 6 } & Female & 189 & 159.3 & 7.6 & 0.50 & \\
\hline
\end{tabular}

In this study, the $\mathrm{CC}$ index (CCI) was estimated by the following formula: $\mathrm{CCI}=($ genu dimension + body dimension + splenium dimension)/APL of the brain. The current findings reported that the mean value of CCI was $0.170 \pm 0.021$. There was a statistically significant gender-related difference for CCI: $0.173 \pm 0.020$ in females and $0.167 \pm 0.021$ in males $(P=0.004)$ (Table 3).

We found a weak but statistically significant indirect correlation between age and CCI $(\mathrm{r}=-0.370, P<0.05)($ Fig 7$)$.

Table 3.

The statistics of CCI in Sudanese participants according to gender

\begin{tabular}{|c|c|c|c|c|c|c|}
\hline Variable & Gender & $\mathrm{n}$ & Mean & SD & SEM & $P$-value \\
\hline \multirow{2}{*}{ CCI } & Male & 196 & 0.167 & 0.021 & 0.0015 & \multirow{2}{*}{0.004} \\
\cline { 2 - 7 } & Female & 189 & 0.173 & 0.020 & 0.0014 & \\
\hline
\end{tabular}

Our results showed a positive correlation between CC length and age. Also, there was a weak direct correlation between age and APL of the brain. Further, the relationship between age and dimensions of CC (genu, body, and splenium) and APL of the brain, CCI, and the CC ratio was presented in Figures 2-8.

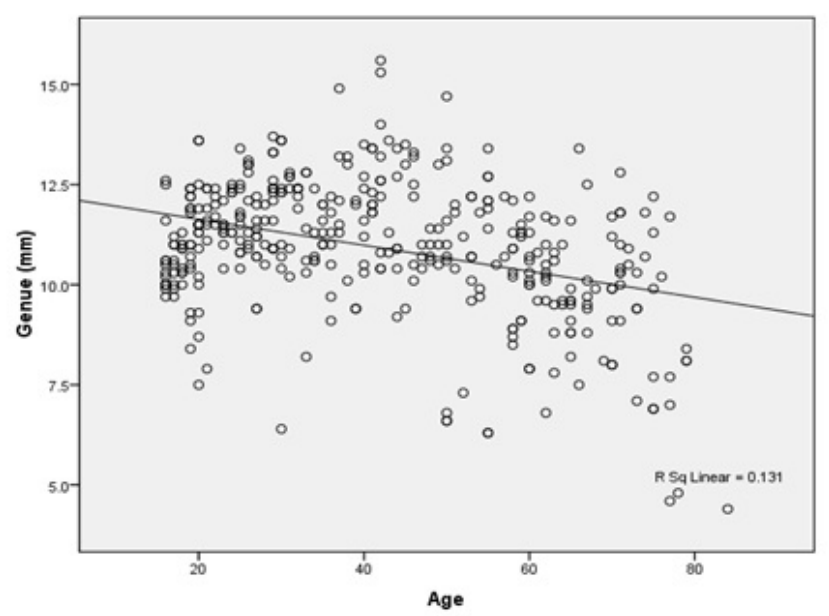

Fig. 2. The relationship between age and the CC genu dimension

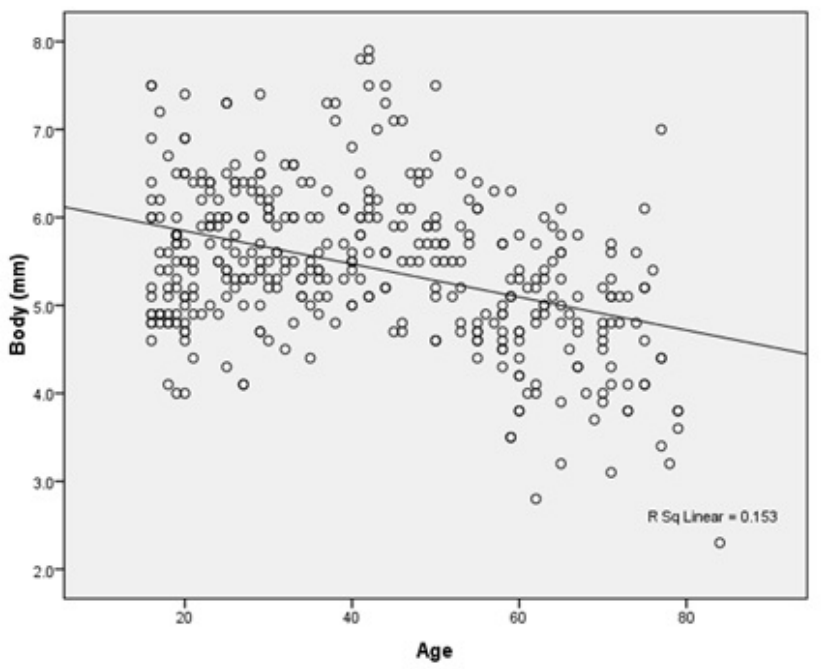

Fig. 3. The relationship between age and the CC body dimension 


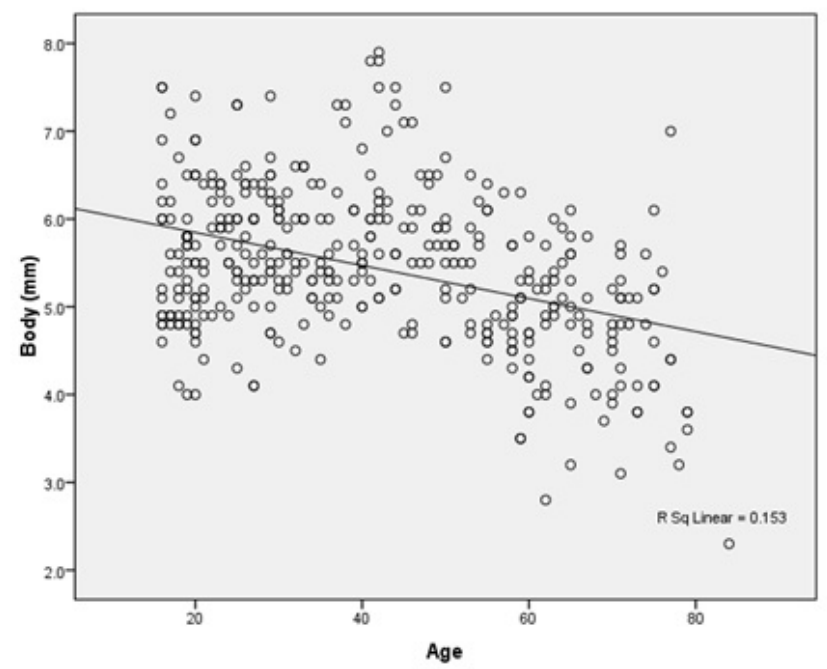

Fig 4. The relationship between age and the CC splenium dimension.

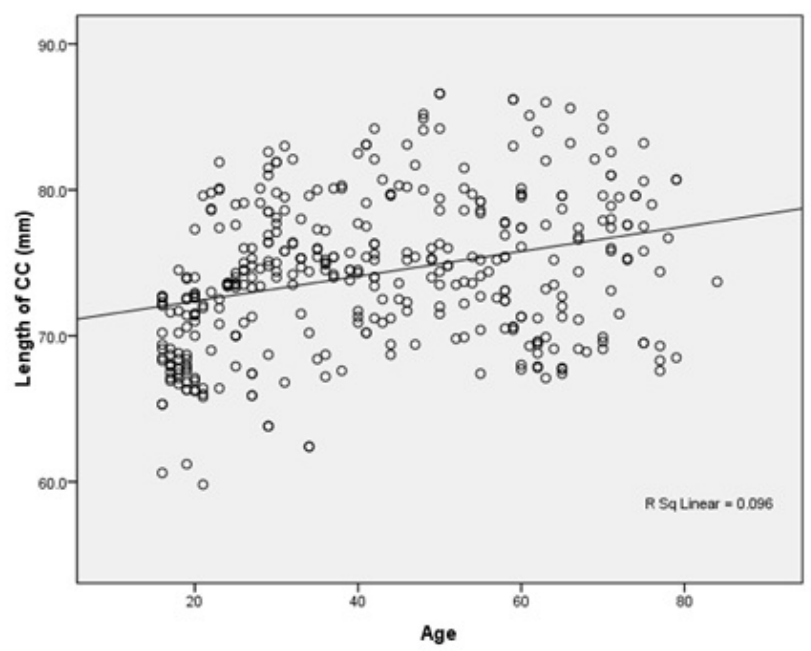

Fig. 5. The relationship between age and the CC length

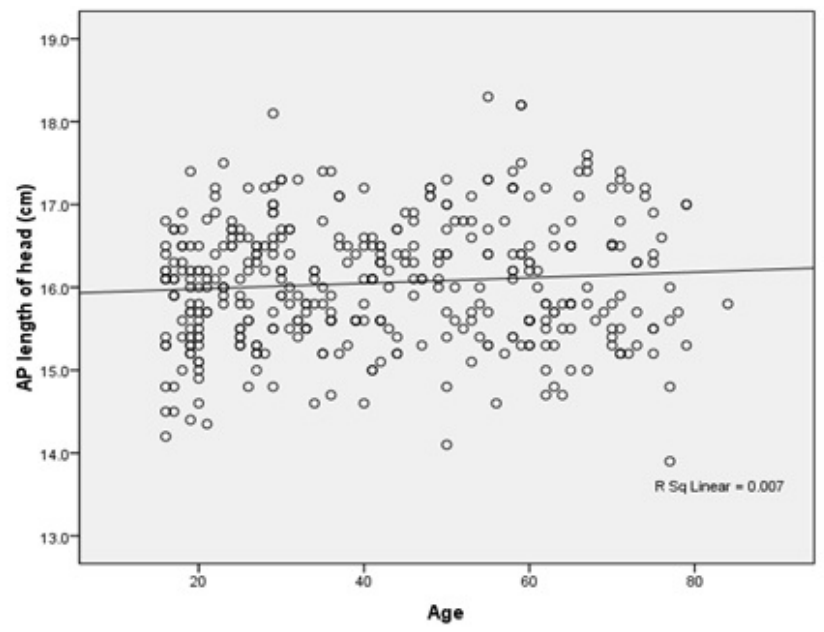

Fig. 6. The relationship between age and APL of the brain.

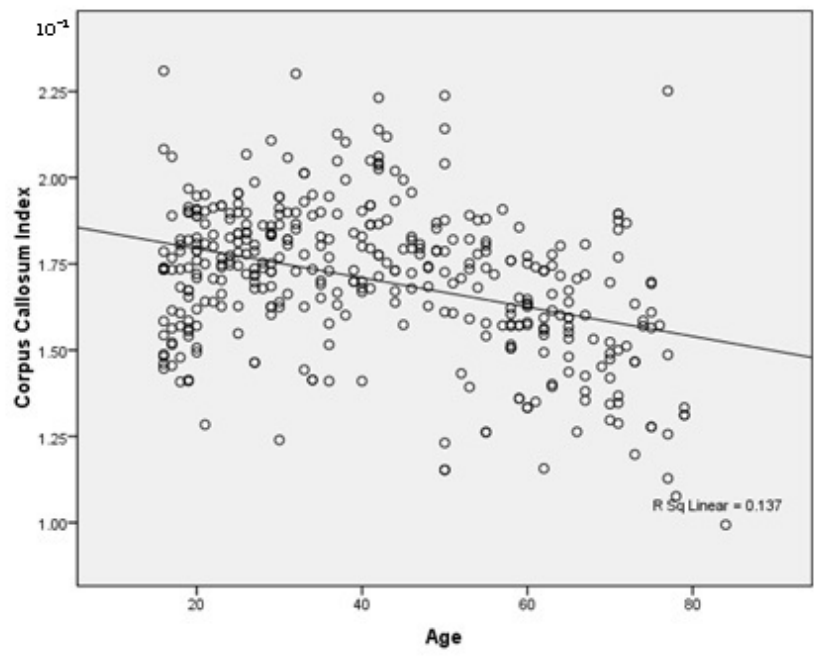

Fig. 7. The relationship between age and CCI.

In this study the $\mathrm{CC}$ ratio was defined as $\mathrm{CC}$ length divided by APL of the brain..$^{(2,9,10)}$ The current findings reported that the mean value of the $C C$ ratio was $0.46 \pm 0.03(0.461 \pm 0.025$ in males and $0.463 \pm 0.03$ in females, $P>0.05$ ) (Table 4). There was a weak but statistically significant direct correlation between age and $\mathrm{CC}$ ratio $(\mathrm{r}=0.271, P<0.05)$ (Fig 8).

Table 4.

The CC ratio statistics according to gender

\begin{tabular}{|c|c|c|c|c|c|c|}
\hline Variable & Gender & $\mathrm{n}$ & Mean & SD & SEM & $P$-value \\
\hline \multirow{2}{*}{ CC ratio } & Male & 196 & 0.461 & 0.025 & 0.001 & \multirow{2}{*}{0.502} \\
\cline { 2 - 7 } & Female & 189 & 0.463 & 0.033 & 0.002 & \\
\hline
\end{tabular}

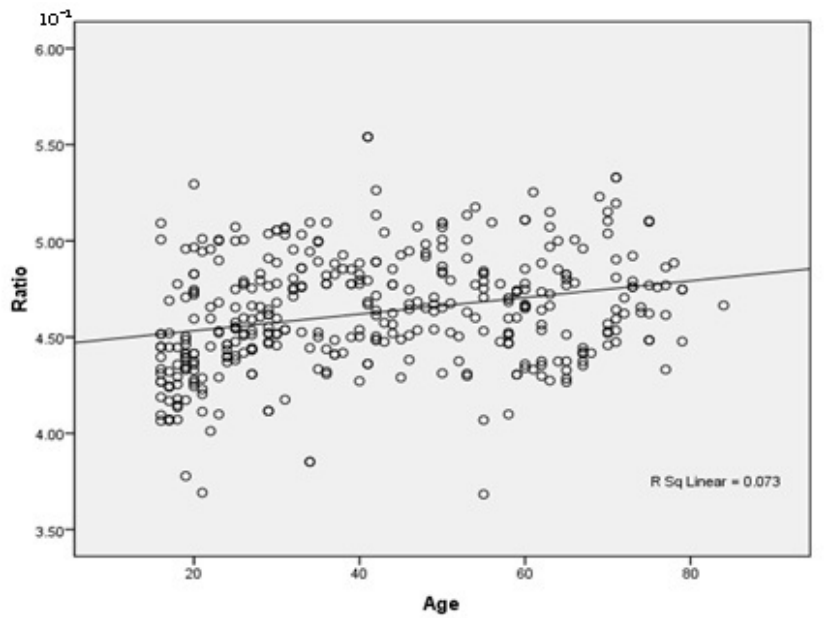

Fig. 8. The relationship between age and CC ratio.

Several MRI studies were conducted to determine the dimensions, morphology, and gender-related differences of $\mathrm{CC}$ in different countries. ${ }^{2,12,13}$ Mourgila et al. ${ }^{(2)}$ found that there was minimal variability in the dimensions and relative 
dimensions of the corpus callosum among all Greek subjects. The longitudinal dimensions of the genu (EZ/3) and total corpus callosum (EZ) were found to be larger in males, whereas the longitudinal dimension of the splenium (EZ/5) was larger in females. Although there was no statistical correlation between corpus callosum and brain dimensions, the authors noted that there was a symmetry between corpus callosum and brain size, with stable proportions, in every studied individual.

For Iranian subjects $(\mathrm{n}=100)$, Mohammadi et al. ${ }^{(9)}$ found that the longitudinal dimensions of $\mathrm{CC}$, genu, and splenium were larger in males than in females, but these differences were not significant. Furthermore APL of the brain was significantly larger in males than in females $(P<0.05)$. The length of $C C$ increased with age and there was also a positive significant correlation between the CC length and APL of the brain.

For Japanese subjects $(\mathrm{n}=205)$, Takeda et al. ${ }^{(12)}$ found that the width of the corpus callosum, including the rostrum, body and splenium, gradually decreased with age. No sexrelated differences in the longitudinal dimensions of $\mathrm{CC}$, including CC length $(69.7 \pm 4.15 \mathrm{~mm}$ in males and $69.4 \pm 4.33$ $\mathrm{mm}$ in females) were observed.

In a study performed by Suganthy et al., ${ }^{(13)}$ the size of the corpus callosum on midsagittal section was measured in 100 (50 males and 50 females) normal adult Indians. The authors reported that the corpus callosum was longer in males and the discriminant score to differentiate gender was determined with an accuracy of $66 \%$; the length of the corpus callosum increased with age.

Bermudez and Zatorre ${ }^{(14)}$ used MRI to measure midsagittal CC area in 137 young normal volunteers. Male subjects showed significantly larger absolute total area, as well as anterior third and posterior midbody. However, females showed relatively larger total area, anterior midbody, and splenium.

For North-West Indian population, Gupta et al. ${ }^{(10)}$ showed that the CC length was $7.57 \mathrm{~cm}$ in males and $7.1 \mathrm{~cm}$ in females; the CC height was $3,27 \mathrm{~cm}$ in males and $2.59 \mathrm{~cm}$ in females. The authors revealed that the Indian values were found to be more than the Japanese values for length, height and most of the widths of CC. The length and width of CC were found to be less than those of Caucasian population.

In another study conducted by Gupta et al. ${ }^{(15)}$, the only CC parameter, which showed sexual dimorphism, was larger $\mathrm{CC}$ length in males.

In the current study, the dimension of the $\mathrm{CC}$ concerning the genu was found to be larger in females in contrast to the findings of Mourgila et al. ${ }^{(2)}$ and Mohammadi et al. ${ }^{(9)}$

A statistically significant gender-related difference for (APL) of the brain found in our study $(159.3 \pm 7.6 \mathrm{~mm}$ in females and $161.6 \pm 7.2 \mathrm{~mm}$ in males $(P=0.002)$ ) (Table 2 ) was in agreement with a previous study by Mohammadi et al. ${ }^{(9)}$ In the current study, we found a weak positive direct correlation between age and APL of the brain. A statistically significant correlation between age and $\mathrm{CC}$ length revealed in the current study (Fig.3) was similar to results obtained by Mohammadi et al. ${ }^{(9)}$ and Suganthy et al..$^{(13)}$

In our study, only the genu length showed sexual dimorphism and was larger in females than in males. A longer corpus callosum in the males has been reported in study by Suganthy et al. ${ }^{(13)}$ Concerning the CC length $(74.5 \pm 4.87 \mathrm{~mm}$ and $73.8 \pm 5.35 \mathrm{~mm}$ in females), our results were higher when compared to studies conducted by Mourgela et al..$^{(2)}$ and Takeda et al. ${ }^{(12)}$, in the other hand these findings were compatible with the results of Gupta et al..$^{(10)}$

The differences in quantitative data of $\mathrm{CC}$ in various areas of the world, which were seen in different studies, might be due to racial/ethnic factors. ${ }^{(2,9,10,15)}$ Further large-scale studies are needed to estimate differences among various ethnicity/races and establish the standard CC dimensions in each population.

In conclusion, the results revealed that only the genu length showed sexual dimorphism and was larger in females than in males. This study showed variabilities regarding the $\mathrm{CC}$ dimensions in Sudanese participants compared to the Caucasian, Indian, and Japanese population.

\section{Acknowledgements}

This publication was supported by the Deanship of Scientific Research at Prince Sattam bin Abdulaziz University, Alkharj, Saudi Arabia.

\section{Competing Interests}

The authors declare that they have no competing interests.

\section{References}

1. Paul LK, Brown WS, Adolphs R, Tyszka JM, Richards LJ, Mukherjee P, Sherr EH. Agenesis of the corpus callosum: genetic, developmental and functional aspects of connectivity. Nat Rev Neurosci. 2007;8(4):287-299. doi:10.1038/nrn2107

2. Mourgela S, Anagnostopoulou S, Sakellaropoulos A, Gouliamos A. An MRI study of sex-and age-related differences in the dimensions of the corpus callosum and brain. Neuroanatomy. 2007;6(1):63-65.

3. Snell RS. Clinical neuroanatomy, 7th Edition. Philidelphia: Lippincott Williams \& Wilkins; 2009.

4. Cignini P, D`emidio L, Padula F, Girgenti A, Battistoni S, Vigna R, Franco R, Rossetti D, Giorlandino M, Giorlandino C. The role of ultrasonography in the diagnosis of fetal isolated complete agenesis of the corpus callosum: a longterm prospective study. J Matern Fetal Neonatal Med. 2010;23(12):1504-1509. doi:10.3109/14767051003678101

5. Keshavan MS, Diwadkar VA, Harenski K, Rosenberg DR, Sweeney JA, Pettegrew JW. Abnormalities of the corpus callosum in first episode, treatment naive schizophrenia. J Neurol Neurosurg Psychiatry. 2002;72(6):757-760. doi:10.1136/ jnnp.72.6.757

*Corresponding author: Professor Mustafa Z. Mahmoud, Radiology and Medical Imaging Department, College of Applied Medical Sciences, Prince Sattam bin Abdulaziz University, E-mail: m.alhassen@psau.edu.sa,PO Box: 422,Zip Code: 11942,Al-Kharj, Saudi Arabia. 
6. Zidan MM, Abdalaziz I, Elnour AM, Alghamdi SS, Mahmoud MZ, Ali WM, Abd-Elgyoum AM. Prevalence and clinical importance of the incidental findings in brain MRI examinations. Bioscience Research. 2020;17(2):888-893.

7. Corballis MC,FinlayDC.Interhemisphericvisualintegration in three cases of familial callosal agenesis. Neuropsychology. 2000;14(1):60-70.

8. Thompson PM, Giedd JN, Woods RP, MacDonald D, Evans AC, Toga AW. Growth patterns in the developing brain detected by using continuum mechanical tensor maps. Nature. 2000;404(6774):190-193. doi:10.1038/35004593

9. Mohammadi MR, Zhand P, Mortazavi Moghadam B, Golalipour MJ. Measurement of the corpus callosum using magnetic resonance imaging in the north of Iran. Iran J Radiol. 2011;8(4):218-223. doi:10.5812/iranjradiol.4495

10. Gupta T, Singh B, Kapoor K, Gupta M, Kochhar S. Normative data of corpus callosal morphology in a NorthWest Indian population- an autopsy and MRI study. JNMA J Nepal Med Assoc. 2009;48(173):46-51.
11. Witelson SF. Hand and sex differences in the isthmus and genu of the human corpus callosum. A postmortem morphological study. Brain. 1989;112(Pt3):799-835. doi:10.1093/brain/112.3.799 12. Takeda S, Hirashima $Y$, Ikeda $H$, Yamamoto $H$, Sugino M, Endo S. Determination of indices of the corpus callosum associated with normal aging in Japanese individuals. Neuroradiology. 2003;45(8):513-518. doi:10.1007/ s00234-003-1019-8

13. Suganthy J, Raghuram L, Antonisamy B, Vettivel S, Madhavi C, Koshi R. Gender- and age-related differences in the morphology of the corpus callosum. Clin Anat. 2003;16(5):396-403. doi:10.1002/ca.10161

14. Bermudez P, Zatorre RJ. Sexual dimorphism in the corpus callosum: methodological considerations in MRI morphometry. Neuroimage. 2001;13(6 Pt 1):1121-1130. doi: 10.1006/nimg.2001.0772

15. Gupta T, Singh B, Kapoor K, Gupta M, Kochhar S. Age and sex related variations in corpus callosal morphology. Nepal Med Coll J. 2008;10(4):215-221. 